\title{
Dynamic Analysis of Coupled Binary Cow Patch Cellular Neural Networks
}

\author{
Min Li, Lequan Min, and Mian Wang
}

\begin{abstract}
Nature abounds with complex patterns emerging from biological, chemical, physical and social systems. Cellular Neural Networks (CNNs) may produce patterns similar to those found in nature, which implies that CNNs may be used as prototypes to describe some systems in nature. The Cow Patch CNNs introduced by Chua et al. can generate pattern that cow patches and checkerboards coexist from any random initial pattern. In order to investigate the characteristics of the Binary Cow Patch CNNs, this study introduces concepts of so-called inherent (final) active, inherent (final) passive, and inherent (final) neutral for pattern pixels, and proposes Global Task and Local Rules of the Binary Cow Patch CNNs, and establishes a set of theorems. Three simulation examples have been carried out to verify the effectiveness of theoretical results.
\end{abstract}

Index Terms-Binary cow patch CNN, initial state, binary image, global task, local rules.

\section{INTRODUCTION}

The CNN introduced by Chua \& Yang ([1], [2]) is a new information processing paradigm. In term of its local connectivity, CNN has been made of CNN universal chips, whose theoretical computation speed is typically three orders of magnitude faster than the current digital processor [3]. Now the CNNs and its generalization have been widely studied for practical applications in image and video signal processing, robotic and biological visions, higher brain functions, and cryptography ( [3]-[15]).

The Hopfield Neural Network (HNN) requires fully connected and grows exponentially with the size of the array. Therefore it cannot be built, even in modest array sizes, as VLSI circuits [3].

In an analog CNN, the parameter levers usually have 5\% $\sim 10 \%$ of perturbation [16]. Therefore, the robust design of $\mathrm{CNN}$ template parameters is an important issue. Chua and Dogaru ([3], [7]) have studied the robust design of a large kind of CNN-uncoupled Boolean CNNs, which provides optimal design schemes for CNNs with prescribed tasks.

Nature abounds with complex patterns and structures emerging from homogeneous media operating far from widely observed in both inanimate (nonbiological) and biological media, can be modeled and studied via the CNN paradigm in an in-depth and unified way [17].

In a Chua's monograph on CNN [3], he has invested

Manuscript received June 1, 2015; revised September 22, 2015. This work was supported in part by the National Natural Science Foundations of China (Grant Nos. 61170037).

The authors are with the School of Mathematics and Physics, University of Science and Technology Beijing, Beijing 100083, PR China (e-mail: limin_mu@126.com, minlequan@sina.com, wangmian14@163.com). spontaneous pattern formations with several kinds of coupled CNNs from random initial conditions. In [18], Thiran, Crounse, Chua, and Hasler have provided a categorization of the patterns that they can formed with $3 \times 3$ template. They have found that for the CNN with "diagonal cross template" (DCT)

$$
A=\left[\begin{array}{lll}
c & 0 & c \\
0 & a & 0 \\
c & 0 & c
\end{array}\right], B=\left[\begin{array}{lll}
0 & 0 & 0 \\
0 & 0 & 0 \\
0 & 0 & 0
\end{array}\right], Z=0
$$

if the parameters satisfy the following inequalities

$$
\begin{gathered}
a+2 c-1>0 \\
a-1<0 \\
c>0,
\end{gathered}
$$

any initial random state input pattern will give rise to stable output pattern that cow patches and checkerboards coexist, which is called the Cow Patch CNN.

In a conference paper [19], Zhang and Min have invested the dynamic behaviors of the stripe $\mathrm{CNN}$ with parameter $a=1$ for any random binary initial input patterns.

In this paper, we introduce a kind of the Binary Cow Patch CNNs. Several theorems are set up to describe the dynamics for the Binary Cow Patch CNNs. Three numerical simulation examples are given to verify the theorems to be efficient in practical applications for computer digital image processing.

The rest of this paper is organized as follows. Section II introduces some definitions related to the behaviors of the Binary Cow Patch CNN, and describes the Global Task and the Local Rules of the Binary Cow Patch CNN. Section III sets up a group of theorems to determine the properties of the output patterns of the Binary Cow Patch CNN. Numerical simulation examples are implemented in Section IV. Finally, Section V gives some conclusions.

\section{BINARY COW PATCH CNN}

In this section, we will show that under conditions (2), (3) and (4), DCT CNN is able to generate output pattern that cow patches and checkerboards coexist from a random initial pattern. First let us remember some basics on CNN.

The standard CNN with $3 \times 3$ template is composed of a two-dimensional $M$ by $N$ array of cells. Each cell is denoted by $C_{i, j}$, where $i=1,2, \cdots, M ; j=1,2, \cdots N$.

The dynamics of $C_{i, j}$ is given by 


$$
\dot{x}_{i, j}=-x_{i, j}+\sum_{k=-1}^{1} \sum_{l=-1}^{1} a_{k, l} y_{i+k, j+l}+\sum_{k=-1}^{1} \sum_{l=-1}^{1} b_{k, l} u_{i+k, j+l}+z
$$

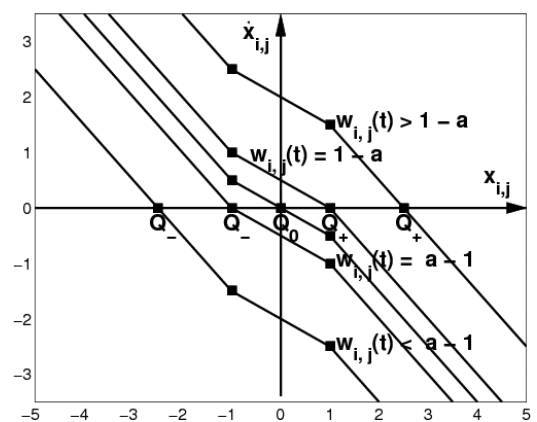

Fig. 1. The dynamic route of Binary Cow Patch CNN with $a<1$.

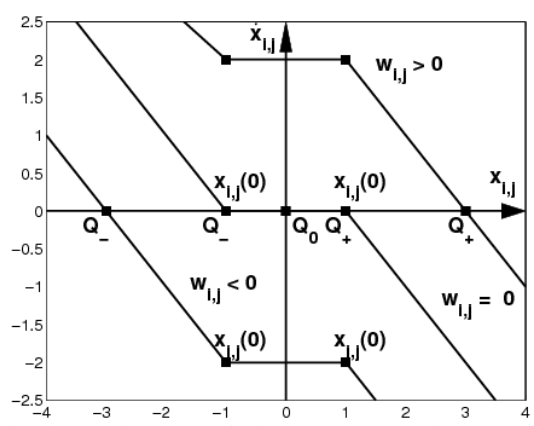

Fig. 2. The dynamic route of Binary Cow Patch CNN with $a=1$.

where $u_{i, j}, x_{i, j}$, and $y_{i, j}$ are the input, state, and output variables of the cell; $a_{k, l}{ }^{\prime} \mathrm{s}, b_{k, l}{ }^{\prime} \mathrm{s}$, and $z$ are the elements of the A-template, the B-template, and threshold, respectively. The output $y_{i, j}$ is the piece-wise linear function given by

$$
y_{i+k, j+l}=\frac{1}{2}\left(\left|x_{i+k, j+l}+1\right|-\left|x_{i+k, j+l}-1\right|\right)
$$
form

The state equation of the Binary Cow Patch CNN has the

$$
\begin{gathered}
\dot{x}_{i, j}(t)=-x_{i, j}(t)+a y_{i, j}(t)+c\left(y_{i+1, j+1}(t)+\right. \\
\left.y_{i-1, j-1}(t)+y_{i+1, j-1}(t)+y_{i-1, j+1}(t)\right) \\
=-x_{i, j}(t)+a y_{i, j}(t)+w_{i, j}(t)
\end{gathered}
$$

The dynamic routes of the Binary Cow Patch CNN are shown in Fig. 1 and Fig. 2. Since the translation term $w_{i, j}$ is dependent on time $t$, this makes the dynamic routes of the Binary Cow Patch CNN are very complex.

In order to describe possible stable equilibria of the Binary Cow Patch CNN, we introduce following definitions.

Definition 1 Let $U=\left(u_{i, j}\right)_{M \times N}$ be a binary image with $M \times N$ pixels. A pixel $C_{i, j}$ at $(i, j)$ th position with gray-scale $u_{i, j}$ is said to be inherent active if

$$
w_{i, j}(t) \geq 1-a, \forall t \geq 0 \text { if } a<1,
$$

or $w_{i, j}(t) \geq 0, \forall t \geq 0$ if $a=1$ and $u_{i, j}=1$, or $w_{i, j}(t)>0, \forall t \geq 0$ if $a=1$ and $u_{i, j}=-1$.
The set consisting of all inherent active pixels is said to be an inherent active network.

A pixel $C_{i, j}$ is said to be inherent passive if

$$
w_{i, j}(t) \leq a-1, \forall t \geq 0 \text { if } a<1,
$$

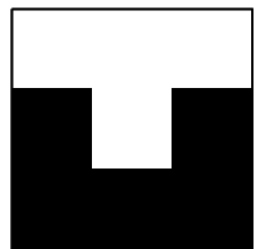

Fig. 3. A pattern $\mathbf{P}$ consists of the inherent passive network and inherent active network.

$$
\begin{aligned}
& \text { or } w_{i, j}(t)<0, \forall t \geq 0 \text { if } a=1 \text { and } u_{i, j}=1, \\
& \text { or } w_{i, j}(t) \leq 0, \forall t \geq 0 \text { if } a=1 \text { and } u_{i, j}=-1
\end{aligned}
$$

The set consisting of all inherent passive pixels is said to be an inherent passive network.

A pixel $C_{i, j}$ is said to be inherent neutral if

$$
a-1<w_{i, j}(t)<1-a, \forall t \geq 0 \text { if } a<1 .
$$

The set consisting of all inherent neutral pixels is said to be an inherent neutral network.

Definition 2 Let $U=\left(u_{i, j}\right)_{M \times N}$ be a binary image with $M \times N$ pixels. A pixel $C_{i, j}$ at $(i, j)$ th position with gray - scale $u_{i, j}$ is said to be final active if there is a constant $t^{*}>0$ such that

$$
w_{i, j}(t) \geq 1-a, \forall t \geq t^{*} \text { if } a<1
$$

or

$$
w_{i, j}(t)>0, \forall t \geq t^{*} \text { if } a=1 \text {. }
$$

The set consisting of all final active pixels is said to be a final active network.

A pixel $C_{i, j}$ is said to be final passive if there is a constant $t^{*}>0$ such that

$$
w_{i, j}(t) \leq a-1, \forall t \geq t^{*} \text { if } a<1
$$

or

$$
w_{i, j}(t)<0, \forall t \geq t^{*} \text { if } a=1 \text {. }
$$

The set consisting of all final passive pixels is said to be a final passive network.

A pixel $C_{i, j}$ is said to be final neutral if there is a constant $t^{*}>0$ such that

$$
a-1<w_{i, j}(t)<1-a, \forall t \geq t^{*} \text { if } a<1,
$$

or

$$
w_{i, j}(t)=0, \forall t \geq t^{*} \text { if } a=1 \text {. }
$$

The set consisting of all final neutral pixels is said to be a final 
neutral network.

Definition 3 Let $U=\left(u_{i, j}\right)_{M \times N}$ be a binary image with $M \times N$ pixels. An active network is the set consisting of the inherent active network and the final active network. A passive network is the set consisting of the inherent passive network and the final passive network. A neutral network is the set consisting of the inherent neutral network and the final neutral network.

Remark 1. The output of a passive network is a white (gray-scale is -1) image. The output of an active network is a black (gray-scale is +1) image. The output of a neutral network is a gray (gray-scale is belong to $[-1,1]$ ) image.

Example 1. Now consider $\mathrm{a}=1$ and the binary image $\mathbf{P}$ shown in Fig. 3, whose gray scales are listed as follows.

$$
P=\left[\begin{array}{lll}
C_{1,1} & C_{1,2} & C_{1,3} \\
C_{2,1} & C_{2,2} & C_{2,3} \\
C_{3,1} & C_{3,2} & C_{3,3}
\end{array}\right]=\left[\begin{array}{ccc}
-1 & -1 & -1 \\
1 & -1 & 1 \\
1 & 1 & 1
\end{array}\right]
$$

Now we investigate the dynamic behaviors of $w_{i, j}(t)^{\prime} \mathrm{s}$ as follows.

$$
\begin{aligned}
& C_{1,1}: w_{1,1}(t)=c\left(u_{0,0}+u_{0,2}+u_{2,0}+y_{2,2}(t)\right) \\
& =c\left(-1-1+1+y_{2,2}(t)\right) \leq 0, t \geq 0 \text {. } \\
& C_{1,3}: w_{1,3}(t)=c\left(u_{0,2}+u_{0,4}+u_{2,4}+y_{2,2}(t)\right) \\
& =c\left(-1-1+1+y_{2,2}(t)\right) \leq 0, t \geq 0 \text {. } \\
& C_{3,1}: w_{3,1}(t)=c\left(u_{2,0}+u_{4,0}+u_{4,2}+y_{2,2}(t)\right) \\
& =c\left(1+1+1+y_{2,2}(t)\right)>0, t \geq 0 \text {. } \\
& C_{3,3}: w_{3,3}(t)=c\left(u_{2,4}+u_{4,2}+u_{4,4}+y_{2,2}(t)\right) \\
& =c\left(1+1+1+y_{2,2}(t)\right)>0, t \geq 0 .
\end{aligned}
$$

It follows that $C_{1,1}, C_{1,3}$ are inherent passive cells and $C_{3,1}$, $C_{3,3}$ are inherent active cells. And we conclude that $C_{2,2}$ is an inherent passive cell. On the other hand,

$$
\begin{aligned}
C_{1,2}: & w_{1,2}(t)=c\left(u_{0,1}+u_{0,3}+y_{2,1}(t)+y_{2,3}(t)\right) \\
& =c\left(-1-1+y_{2,1}(t)+y_{2,3}(t)\right) \leq 0, t \geq 0 . \\
C_{3,2}: & w_{3,2}(t)=c\left(u_{4,1}+u_{4,3}+y_{2,1}(t)+y_{2,3}(t)\right) \\
& =c\left(1+1+y_{2,1}(t)+y_{2,3}(t)\right) \geq 0, t \geq 0 .
\end{aligned}
$$

It follows that $C_{1,2}$ is an inherent passive cell and $C_{3,2}$ is an inherent active cell.

$$
\begin{gathered}
C_{2,1}: w_{2,1}(t)=c\left(u_{1,0}+u_{3,0}+y_{1,2}(t)+y_{3,2}(t)\right) \\
=c\left(-1+1+y_{1,2}(t)+y_{3,2}(t)\right)=0, t \geq 0 . \\
C_{2,3}: w_{2,3}(t)=c\left(u_{1,4}+u_{3,4}+y_{1,2}(t)+y_{3,2}(t)\right) \\
=c\left(-1+1+y_{1,2}(t)+y_{3,2}(t)\right)=0, t \geq 0 .
\end{gathered}
$$

It follows that $C_{2,1}$ and $C_{2,3}$ are inherent active cells.
Therefore $C_{1,1}, C_{1,2}, C_{1,3}$ and $C_{2,2}$ are consist of inherent passive network. $C_{2,1}, C_{2,3}, C_{3,1}, C_{3,2}$ and $C_{3,3}$ are consist of inherent active networks.

In order to describe the stable output images of Binary Cow Patch CNN, we introduce following definitions.

Definition 4 A cow patch black/white island is the cow patch-shaped area. This area is composed of black/white pixels connected by edges, and every black/white pixel has at least three black/white pixels connected with it.

Remark 2. If an initial state $X(0)=U$. Then the stable output image of a cow patch black /white for a Binary Cow Patch CNN is the same cow patch-shaped black/white island.

Definition 5 A checkerboard black/white island is the checkerboard-shaped area. This area is composed of black/white pixels connected diagonally and every black/white pixel has at least two black/white pixels connected with it.
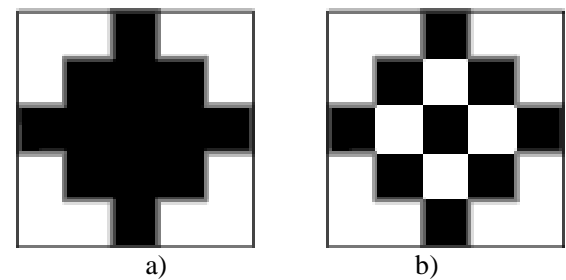

Fig. 4. a) A Cow Patch black island; b) A Checkerboard black island.

Remark 3. If an initial state $X(0)=U$. Then the stable output image of a checkerboard black/white for a Binary Cow Patch CNN is the same checkerboard-shaped black/white island.

Example 2. The area marked in Fig. 4 a) is a cow patch black island, and the area marked in Fig. 4 b) is a checkerboard black island.

Now we propose the Global Task and Local Rules for the Binary Cow Patch CNN.

\section{A. Global Task of the Binary Cow Patch CNN}

1) Given: A static binary image $U$.

2) Initial State: $X(0)=U$.

3) Boundary Conditions: State variable is reflected across the boundary.

4) Output: $Y(t) \Rightarrow Y(\infty)$ consists of pattern that cow patches and checkerboards coexist [18].

B. Local Rules of the Binary Cow Patch CNN

$$
u_{i, j} \quad y_{i, j}(\infty) .
$$

white / black $\rightarrow$ black, if $C_{i, j}$ is inherent active or final active. white / black $\rightarrow$ white, if $C_{i, j}$ is inherent passive or final passive. white / black $\rightarrow$ gray, if $C_{i, j}$ is inherent neutral or final neutral.

\section{MATHEMATICAL ANALYSIS}

In 1999, Lin and Shih showed the following: 
Theorem 1: ([20]) Any Standard CNN with symmetric space-variant feedback template is completely stable

It follows that each CNN with diagonal cross template is complete stable, that is, has a stable state output for any initial condition.

From Fig. 1, we can conclude that:

$$
y_{i, j}(\infty)=\left\{\begin{array}{cc}
1 & \text { if } w_{i, j}(t) \geq 1-a, \forall t \geq 0 ; \\
-1 & \text { if } w_{i, j} \leq a-1, \forall t \geq 0 ; \\
\text { gray } & \text { if } a-1<w_{i, j}(t)<1-a, \forall t \geq 0 ; \\
1 & \text { if there exists a constant } t^{*}>0, \\
& \text { such that } w_{i, j}(t) \geq 1-a, \forall t \geq t^{*} ; \\
-1 \quad & \text { if there exists a constant } t^{*}>0, \\
& \text { such that } w_{i, j}(t) \leq a-1, \forall t \geq t^{*} ; \\
\text { if there exists } a \text { constant } t^{*}>0, \\
\text { such that } a-1<w_{i, j}(t)<1-a, \\
\forall t \geq t^{*} .
\end{array}\right.
$$

From Fig. 2, we can conclude that:

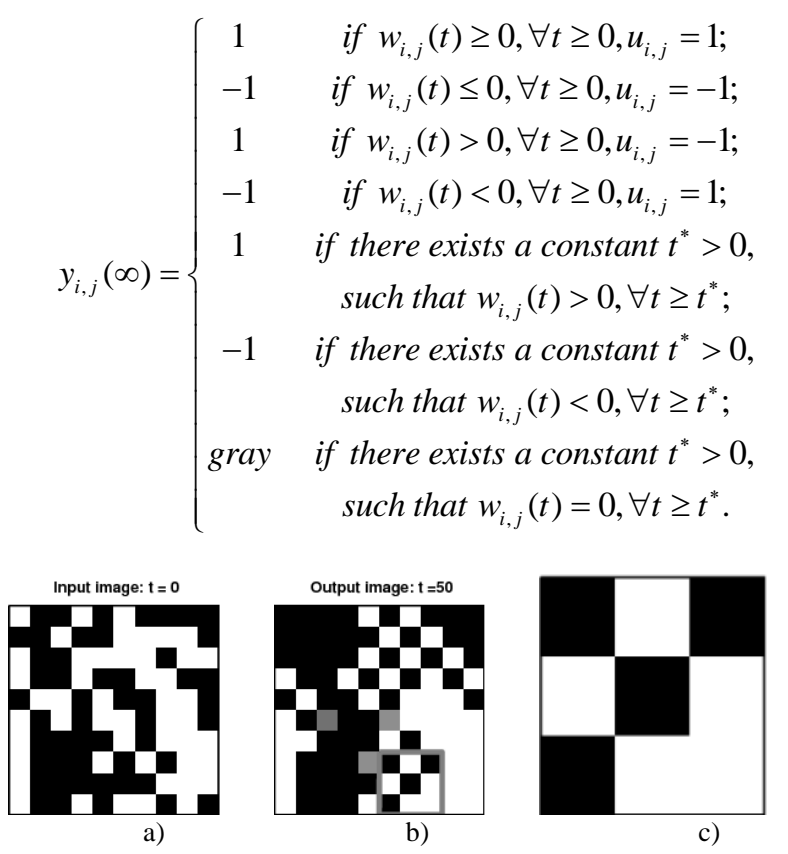

Fig. 5. a) Input image; b) Output image; c) Image selected from

Fig. 5 a) is a random input binary image, Fig. 5(b) is the stable output image by the Binary Cow Patch CNN, Fig. 5(c) is an extracted area marked in Fig. 5 b).

The following theorem gives necessary conditions to make the Binary Cow Patch CNN has the output shown in Fig. 5 b).

Theorem 2: Let the positions of CNN template parameters be described by (1). If a CNN can generate cow patch-shaped pattern from a random binary initial pattern then the following parameter inequality must hold,

$$
\begin{aligned}
& a+2 c-1 \geq 0 \\
& a-1 \leq 0 \\
& c>0
\end{aligned}
$$

Proof: If the stable output images of the CNN has a pattern as shown in Fig. 5 c). According to Local Rule 1, formulas (7) and (8), for cell $C_{2,2}$ should meet

$$
\begin{aligned}
w_{2,2}(\infty) & =c\left(y_{1,1}(\infty)+y_{1,3}(\infty)+y_{3,1}(\infty)+y_{3,3}(\infty)\right) \\
& =c(1+1+1-1)=2 c \geq 1-a, \text { if } a<1, \\
w_{2,2}(\infty) & =c\left(y_{1,1}(\infty)+y_{1,3}(\infty)+y_{3,1}(\infty)+y_{3,3}(\infty)\right) \\
& =c(1+1+1-1)=2 c>0, \text { if } a=1 .
\end{aligned}
$$

Hence formulas (9), (10) and (11) must hold.

We present two theorems on inherent active network and inherent passive network.

Theorem 3: A pixel $C_{i, j}$ is inherent active if there are at least three nearest fixed black neighbors on its diagonal lines.

Proof: By there are at least three nearest fixed black neighbors on its diagonal lines, therefore,

$$
w_{i, j}(t) \geq c(3-1)=2 c \geq 1-a, \forall t \geq 0 \text { if } a<1,
$$

or

$$
w_{i, j}(t) \geq c(3-1)=2 c>0, \forall t \geq 0 \text { if } a=1 .
$$

From definition 1, it follows that $C_{i, j}$ is inherent active.

Theorem 4: A pixel $C_{i, j}$ is inherent passive if there are at least three nearest fixed white neighbors on its diagonal lines.

Proof: By there are at least three nearest fixed white neighbors on its diagonal lines, therefore,

$$
w_{i, j}(t) \leq c(-3+1)=-2 c \leq a-1, \forall t \geq 0 \text { if } a<1,
$$

or

$$
w_{i, j}(t) \leq c(-3+1)=-2 c<0, \forall t \geq 0 \text { if } a=1 .
$$

From definition 1, it follows that $C_{i, j}$ is inherent passive.

Then, we present two theorems about final active network, final passive network and final neutral network.

Theorem 5: Let a pixel $C_{i, j}$ neither be inherent active nor inherent passive. Then

1) if there are at least three nearest diagonal neighbors are inherent active, then $C_{i, j}$ is final active. That is, $y_{i, j}(\infty)=1$.

2) if there are at least three nearest diagonal neighbors are inherent passive, then $C_{i, j}$ is final passive. That is, $y_{i, j}(\infty)=-1$.

$3)$ if there are exactly two nearest diagonal neighbors are inherent active and two nearest diagonal neighbors are inherent passive, then $C_{i, j}$ is final neutral. That is, there exists a constant $t^{*}>0$ such that $y_{i, j}(t)=x_{i, j}\left(t^{*}\right), \forall t \geq t^{*}$

Proof:

1) By the Definition of the inherent active pixels and Local Rule 1 , there is a constant $t^{*}>0$ such that $\forall t \geq t^{*}$, there are at least three nearest diagonal neighbors become black. Therefore, 


$$
w_{i, j}(t) \geq c(3-1)=2 c \geq 1-a, \forall t \geq t^{*} \text { if } a<1,
$$

or

$$
w_{i, j}(t) \geq c(3-1)=2 c>0, \forall t \geq t^{*} \text { if } a=1 .
$$

By Definition 2, it follows that $C_{i, j}$ is final active.

2) By the Definition of the inherent passive pixels and Local Rule 2, there is a constant $t^{*}>0$ such that $\forall t \geq t^{*}$, there are at least three nearest diagonal neighbors become white. Therefore,

$$
w_{i, j}(t) \leq c(-3+1)=-2 c \leq a-1, \forall t \geq t^{*} \text { if } a<1,
$$

or

$$
w_{i, j}(t) \leq c(-3+1)=-2 c<0, \forall t \geq t^{*} \text { if } a=1 .
$$

By Definition 2, it follows that $C_{i, j}$ is final passive.

3) By the Definition of the inherent active, passive pixels and Local Rule 3, there is a constant $t^{*}>0$ such that $\forall t \geq t^{*}$, there are two nearest diagonal neighbors become black and two become white. Therefore,

$$
w_{i, j}(t)=c(2-2)=0, \forall t \geq t^{*} \text { if } a \leq 1 .
$$

By Definition 2, it follows that $C_{i, j}$ is final neutral.

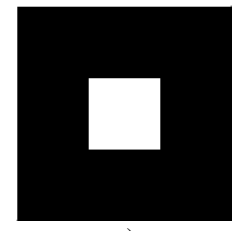

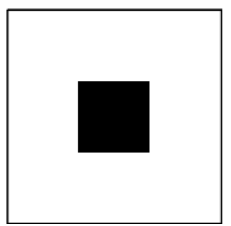

b)
Fig. 6. a) White pixel in isolated way; b) Black pixel in isolated way.

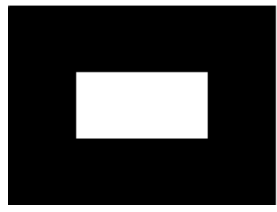

a)

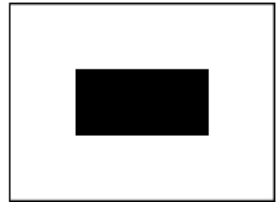

b)
Fig. 7. a) Two white pixels connected by edge in isolated way; b) Two black pixels connected by edge in isolated way.

Theorem 6: Let a pixel $C_{i, j}$ neither be inherent active nor inherent passive. If there is a constant $t^{*}>0$ such that $\forall t \geq t^{*}$

$$
\begin{aligned}
& \text { 1) } w_{i, j}(t) \geq 1-a \text { or } w_{i, j}(t)>0 \text {, then } y_{i, j}(\infty)=1, \\
& \text { 2) } w_{i, j}(t) \leq a-1 \text { or } w_{i, j}(t)<0 \text {, then } y_{i, j}(\infty)=-1, \\
& \text { 3) } a-1<w_{i, j}(t)<1-\text { a or } w_{i, j}(t)=0 \text {, then } y_{i, j}(\infty)=x_{i, j}\left(t^{*}\right)
\end{aligned}
$$

Proof: It can be concluded from Local Rules, formulas (7) and (8).

In the following discussions, we will present several theorems and corollaries on the properties of the output images processed by the Binary Cow Patch CNN.

Theorem 7: For any random binary initial input image, the stable output image of the Binary Cow Patch CNN does not have the following properties:

1) An white/black pixel (see Fig. 6) exists in isolated way.

2) Two white/black pixels connected by edge (see Fig. 7) exist in isolated ways.

Proof:

1) For cell $C_{2,2}$ in Fig. 6 a),

$$
\begin{aligned}
w_{2,2}(\infty) & =c\left(y_{1,1}(\infty)+y_{1,3}(\infty)+y_{3,1}(\infty)+y_{3,3}(\infty)\right) \\
& =c(1+1+1+1) \\
& =4 c>2 c \geq 1-a, \text { if } a<1,
\end{aligned}
$$

$$
\begin{aligned}
w_{2,2}(\infty) & =c\left(y_{1,1}(\infty)+y_{1,3}(\infty)+y_{3,1}(\infty)+y_{3,3}(\infty)\right) \\
& =c(1+1+1+1)=4 c>0, \text { if } a=1 .
\end{aligned}
$$

Hence the stable output gray-level of the cell $C_{2,2}$ must be black. Similarly, the stable output gray-level of cell $C_{2,2}$ in Fig. 6 b) should be white.

2) For cell $C_{2,2}$ in Fig. 7 a),

$$
\begin{aligned}
w_{2,2}(\infty) & =c\left(y_{1,1}(\infty)+y_{1,3}(\infty)+y_{3,1}(\infty)+y_{3,3}(\infty)\right) \\
& =c(1+1+1+1) \\
& =4 c>2 c \geq 1-a, \text { if } a<1, \\
w_{2,2}(\infty) & =c\left(y_{1,1}(\infty)+y_{1,3}(\infty)+y_{3,1}(\infty)+y_{3,3}(\infty)\right) \\
& =c(1+1+1+1)=4 c>0, \text { if } a=1 .
\end{aligned}
$$

Hence the stable output gray-level of the cell $C_{2,2}$ must be black. Similarly, the stable output gray-level of cell $C_{2,3}$ in Fig. 7 a) should be black, and the stable output gray-level of cells $C_{2,2}, C_{2,3}$ in Fig. 7 b) should be white.

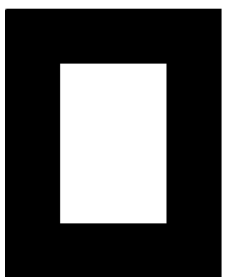

a)

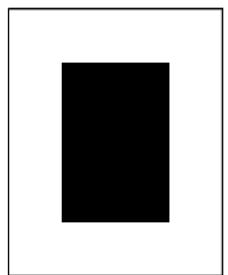

b)
Fig. 8. a) Two pixels wide white striped band which are connected by edges in isolated way; b) Two pixels wide black striped band which are connected by edges in isolated way.

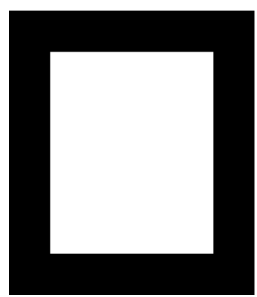

a)

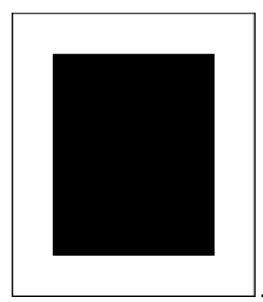

b)
Fig. 9. a) White rectangle in isolated way; b) Black rectangle in isolated way

Corollary 1: For any random initial input image, horizontal or vertical lines consisting of white/black pixels which connected by edge in isolated way cannot exist as the stable output image of the Binary Cow Patch CNN.

Theorem 8: For any random binary initial input image, the 
stable output image of the Binary Cow Patch CNN does not have the following properties:

1) Two pixels wide striped bands which are connected by edges (see Fig. 8) exist in isolated ways.

2) Rectangle/square white/black blocks which are connected by edges (see Fig. 9) exist in isolated ways.

Proof:

1) For cell $C_{2,2}$ in Fig. 8 a),

$$
\begin{aligned}
w_{2,2}(\infty) & =c\left(y_{1,1}(\infty)+y_{1,3}(\infty)+y_{3,1}(\infty)+y_{3,3}(\infty)\right) \\
& =c(1+1+1-1) \\
& =2 c \geq 1-a, \text { if } a<1, \\
w_{2,2}(\infty) & =c\left(y_{1,1}(\infty)+y_{1,3}(\infty)+y_{3,1}(\infty)+y_{3,3}(\infty)\right) \\
& =c(1+1+1-1)=2 c>0, \text { if } a=1 .
\end{aligned}
$$

Hence the stable output gray-level of the cell $C_{2,2}$ must be black. Similarly, the stable output gray-level of cells $C_{2,3}, C_{4,2}, C_{4,3}, C_{3,2}, C_{3,3}$ in Fig. 8 a) should be black, and the stable output gray-level of cells $C_{2,2}, C_{2,3}, C_{4,2}, C_{4,3}, C_{3,2}, C_{3,3}$ in Fig. 8 b) should be white.

2) For $C_{2,2}$ in Fig. 9 a),

$$
\begin{aligned}
w_{2,2}(\infty) & =c\left(y_{1,1}(\infty)+y_{1,3}(\infty)+y_{3,1}(\infty)+y_{3,3}(\infty)\right) \\
& =c(1+1+1-1) \\
& =2 c \geq 1-a, \text { if } a<1, \\
w_{2,2}(\infty) & =c\left(y_{1,1}(\infty)+y_{1,3}(\infty)+y_{3,1}(\infty)+y_{3,3}(\infty)\right) \\
& =c(1+1+1-1)=2 c>0, \text { if } a=1 .
\end{aligned}
$$

Hence the stable output gray-level of the cell $C_{2,2}$ must be black. Similarly, the stable output gray-level of cells

$C_{2,5}, C_{6,2}, C_{6,5}$ in Fig. 9 a) should be black, and the stable output gray-level of cells $C_{2,2}, C_{2,5}, C_{6,2}, C_{6,5}$ in Fig. 9 b) should be white.

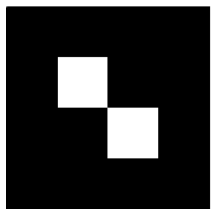

a)

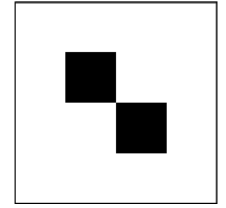

b)
Fig. 10. a) Two white pixels diagonally connected pattern in isolated way; b) Two black pixels diagonally connected pattern in isolated way.

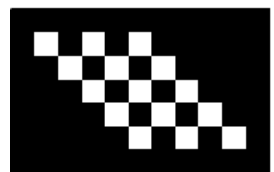

a)

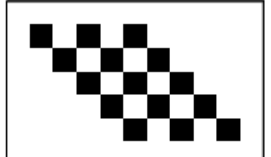

b)
Fig. 11. a) White blocks which are connected by diagonal points; b) Black blocks which are connected by diagonal points.

Theorem 9: For any random binary initial input image, two white/black pixels diagonally connected pattern (see Fig. 10) in isolated ways cannot exist as the stable output image generated by the Binary Cow Patch CNN.

Proof: For cell $C_{2,2}$ in Fig. 10 a),

$$
\begin{aligned}
w_{2,2}(\infty) & =c\left(y_{1,1}(\infty)+y_{1,3}(\infty)+y_{3,1}(\infty)+y_{3,3}(\infty)\right) \\
& =c(1+1+1-1) \\
& =2 c \geq 1-a, \text { if } a<1, \\
w_{2,2}(\infty) & =c\left(y_{1,1}(\infty)+y_{1,3}(\infty)+y_{3,1}(\infty)+y_{3,3}(\infty)\right) \\
& =c(1+1+1-1)=2 c>0, \text { if } a=1 .
\end{aligned}
$$

Hence the stable output gray-level of the cell $C_{2,2}$ must be black. Similarly, the stable output gray-level of cell $C_{3,3}$ in Fig. 10 a) should be black, and the stable output gray-level of cells $C_{2,2}, C_{3,3}$ in Fig. 10 b) should be white.

Corollary 2: For any random initial input image, diagonal lines consisting of white/black pixels which diagonally connected in isolated ways cannot exist as the stable output image of the Gray Cow Patches and Checkerboards coexist CNN.

Theorem 10: For any random binary initial input image, white/black blocks which are connected by diagonal points (see Fig. 11) in isolated ways cannot exist as the stable output image generated by the Binary Cow Patch CNN.

Proof: For cell $C_{2,2}$ in Fig. 11 a),

$$
\begin{aligned}
w_{2,2}(\infty) & =c\left(y_{1,1}(\infty)+y_{1,3}(\infty)+y_{3,1}(\infty)+y_{3,3}(\infty)\right) \\
& =c(1+1+1-1) \\
& =2 c \geq 1-a, \text { if } a<1, \\
w_{2,2}(\infty) & =c\left(y_{1,1}(\infty)+y_{1,3}(\infty)+y_{3,1}(\infty)+y_{3,3}(\infty)\right) \\
& =c(1+1+1-1)=2 c>0, \text { if } a=1 .
\end{aligned}
$$

Hence the stable output gray-level of the cell $C_{2,2}$ must be black. Similarly, the stable output gray-level of cell $C_{6,10}$ in Fig. 11 a) should be black, and the stable output gray-level of cells $C_{2,2}, C_{6,10}$ in Fig. 11 b) should be white.

In summary, we complete the proof.

Remark 4. For any $a+2 c-1 \geq 0, a \leq 1, c>0$ and any random binary input image, the output of the Binary Cow Patch CNN is the same, that is, the performance of the Binary Cow Patch CNN is robust.

\section{NUMERICAL SIMULATION}

In this section, we will provide three experimental simulation examples in three initial states for designed Binary Cow Patch CNN.

Now let us consider the following Binary Cow Patch CNN template for simulation.

$$
A=\left[\begin{array}{ccc}
0.2 & 0 & 0.2 \\
0 & 1 & 0 \\
0.2 & 0 & 0.2
\end{array}\right], B=\left[\begin{array}{lll}
0 & 0 & 0 \\
0 & 0 & 0 \\
0 & 0 & 0
\end{array}\right], Z=0
$$

Fig. 12 a) - Fig. 12 c) are random binary images with pixels generated via the Matlab command $2 *(\operatorname{randi}([0 \quad 1], 60,60)-0.5)$ 
Fig. 12 d) - Fig. 12 g) are the corresponding output images generated via the Binary Cow Patch CNN with template (12).

Observe that although inputs are three different random binary images, the output images show similar cow patch-shaped and checkerboard-shaped patterns, as the theories predict.

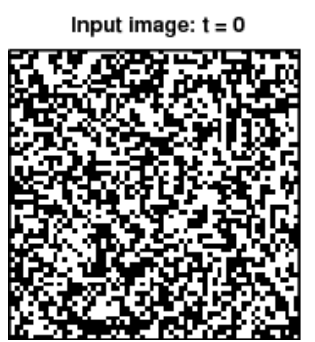

a)

Input image: $\mathrm{t}=0$

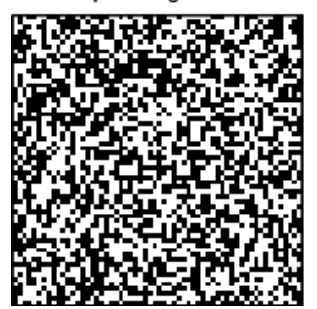

b)

Input image: $t=0$

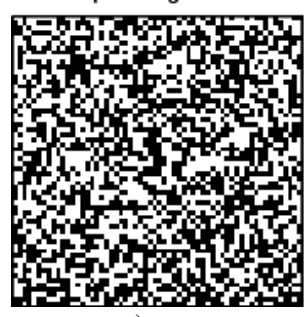

c)
Output image: $\mathrm{t}=\mathbf{2 0 0}$

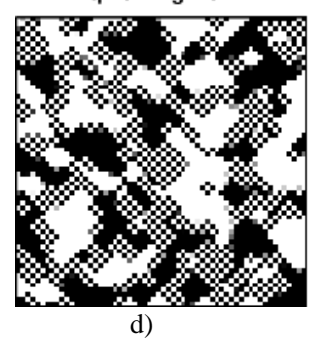

Output image: $t=200$

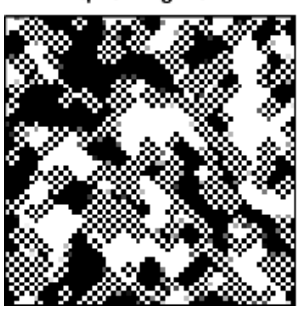

e)

Output image: $t=200$

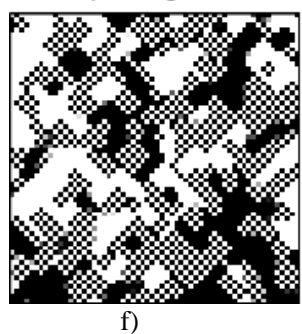

f)
Fig. 12. The inputs and outputs of three simulation examples.

\section{CONCLUSIONS}

This paper investigates a kind of Binary Cow Patch CNNs. Ten theorems and two corollaries describe the main characteristics of the output images for the Binary Cow Patch CNNs, and prove.

This study introduces the definitions of inherent (final) active network, inherent (final) passive network, and inherent (final) neutral network. These definitions have significant biological meanings. Practically, we are difficult to confirm whether a cell is inherent (final) active, inherent (final) passive, or inherent (final) neutral. However, these concepts suggest that for a specific Binary Cow Patch CNN, there is a threshold interval (or the threshold 0 in the case that the template parameter $a=1$ ) such that the pixels as output pattern of the Binary Cow Patch CNN are classified three kinds:

1) Active networks. They may represent a kind specific substance, or specific pattern that cow patches and checkerboards coexist or normal (uninfected or undamaged) cells.

2) Passive networks. They may represent another kind specific substance, or another specific pattern that cow patches and checkerboards coexist or abnormal (infected or damaged) cells.
3) Neutral networks. They may represent interim substances, or interim pattern that cow patches and checkerboards coexist or interim (infecting or damaging) cells.

The three simulation examples confirm that the theoretical analysis is efficient in practical applications for computer numerical simulations.

The dynamic behaviors of other kind CNNs with diagonal cross templates are also quite interesting ([3], [18]).

The further researches on those CNNs may be helpful for interpreting or describing some physical, chemical, biological and social phenomena.

\section{ACKNOWLEDGMENT}

L. Min would like to express since gratitude to professor Leon O. Chua for directing him to study the fascinating CNN.

\section{REFERENCES}

[1] L. O. Chua and L. Yang, "Cellular neural networks: Theory," IEEE Trans. Circuits Syst., vol. 35, pp. 1257-1272, 1988.

[2] IEEE Trans. Circuits Syst., vol. 35, pp. 1273-1290, 1988.

[3] L. O. Chua, "CNN: A version of complexity," Int. J. Bifurcation and Chaos, vol. 7, no. 10, pp. 2219-2425, 1997.

[4] IEEE Transactions on Circuit and Systems I, vol. 42, no. 10, 1995.

[5] L. Orzo, Z. Vidnyanszky, J. Hamori, and T. Roska, "Cnn model of the feature-linked synchronized activities in the visual thalamo-cortical system," Workshop on Cellular Neural Networks and Their Application (CNNA-96), Seville, Spain, pp. 1257-1272, 1988.

[6] F. Weblin, A. Jacobs, and J. Teeters, "The computational eye," IEEE Spectrum, pp. 30-37, 1996.

[7] R. Dogaru and L. O. Chua, "Universal CNN cells," International Journal of Bifurcation of Chaos, vol. 9, pp. 1-48,1999.

[8] L. O. Chua and T. Roska, Cellular Neural Networks and Visual Computing, Cambridge: Cambridge University Press, 2002.

[9] P. Arena, A. Basile, L. Fortuna, and M. Frasca, "CNN wave based computation for robot navigation," in Proc. Int. Symposium on Circuit and Systems, vol. 5, pp. 500-503, 2004.

[10] J. Liu and L. Min, "Robust designs for gray-scale global connectivity detection cnn template," Int. J. Bifurc. Chaos, vol. 17, no. 8, pp. 2827-2838., 2007.

[11] G. Li, L. Min, and H. Zang, "Color edge detections based on cellular neural network," International Journal of Bifurcation and Chaos, vol. 18, no.4, pp. 1231-1242, 2008.

[12] H. Zang and L. Min, "Generalized synchronization theorems for a kind of neural network with application in data encryption," in Proc. 3rd IEEE Conference on Industrial Electronics and Applications, Singapore, 2008, pp. 948-953.

[13] H. Babatunde, O. Folorunso, and A. Akinwale, "A cellular neural network-based model for edge detection," Journal of Information and Computing Science, vol. 5, no. 1, pp. 003-010, 2010.

[14] J. Liu and L. Min, "Robust design of bipolar wave cellular neural network with applications," International Journal of Modeling, Identification and Control, vol. 11, no. 1/2, pp. 99-106, 2010.

[15] J. Li, F. Bai, and X. Di, "New color image encryption algorithm based on compound chaos mapping and hyperchaotic cellular neural network," Journal of Electronic Imaging, vol. 22, no. 1, pp. 013 036-013 036, 2013.

[16] M. Hanggi and G. S. Moschytz, "Genetic optimization of cellular neural networks," in Proc. IEEE Int. Conf. Evolutionary Computation, Anchorage, AK, 1988, pp. 381-386.

[17] L. O. Chua, "Passivity and complexity," IEEE Transactions on Circuit and Systems I, vol. 46, no. 1, pp. 72-82, 1999.

[18] P. Thiran, K. R. Crounse, L. O. Chua, and M. Hasler, "Pattern formation properties of autonomous cellular neural networks," IEEE Transactions on Circuit and Systems I, vol. 42, no. 10, pp. 757-774, 1995.

[19] Q. Zhang and L. Min, "Dynamic analysis of coupled binary stripe CNNS," in Proc. the 2011 IEEE International Conference on Intelligent Computation and Bio-medical Instrumentation, 2011, pp. 236-240.

[20] S. Lin and C. Shih, "Complete stability for stadand cellular neural networks," International Journal of Bifurcation and Chaos, vol. 9, no. 5, pp. 909-918, 1999. 
Min $\mathbf{L i}$ is currently a master science candidate of School of Mathematics and Physics at the University of Science and Technology Beijing. Her current research interest is in the area of cellular neural networks, image analysis and image processing.

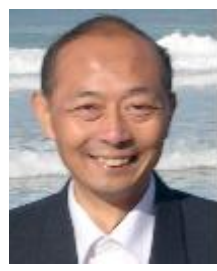

Lequan Min is currently a professor of the Department of Information and Computer Sciences, School of Mathematics and Physics at the University of Science and Technology Beijing. His current research interest is in the areas of image processing, chaos generalized synchronization and modeling virus infection dynamics. He is the author and coauthor of over three hundred scientific papers.

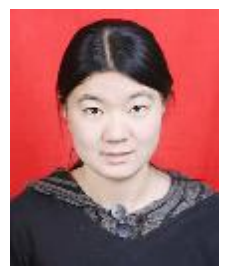

Mian Wang is currently a master science candidate of School of Mathematics and Physics at the University of Science and Technology Beijing. Her current research interest is in the area of cellular neural networks and image analysis. 